\title{
Transfer Function Based Modeling of the Air-Cored Transformers for Very Fast Transient Overvoltage Studies
}

\author{
E. Agheb, S. Esmaillzadeh, K. Niayesh, J. Jadidian and E. Hashemi \\ School of Electrical and Computer Engineering, IR-143950, Tehran University, Iran
}

\begin{abstract}
In this paper, to calculate overvoltage in air-cored transformer windings under very fast transient overvoltage, firstly, the voltage transfer functions are calculated which are measured by network analyser or calculated from the distributed parameters of transformer windings. Secondly, the voltage transfer functions are fitted with rational functions by vector fitting codes and then the rational transfer functions are order-reduced by the Pade approximation. Lastly, the resultant voltage transfer functions are synthesized by the elements in Electromagnetic Transients program. The calculated voltages from the circuit by Electromagnetic Transients program are in good agreement with the measured voltages. This shows that the method is simple and robust.
\end{abstract}

PACS numbers: 52.90.+z

\section{Introduction}

Accurate representation of physical systems by a transfer function is often needed in various fields of engineering for purposes of analysis, design, or simulation. System identification by terminal characteristics may also be useful for the lumped parameter modelling of distributed systems, reduction of model order, and simplification of complex systems [1]. In the pulsed power engineering area, wide frequency range modelling of transformers and reactors by frequency domain external measurements is sometimes required for the study of electromagnetic transients. Besides, the "black-box" representation of such equipment in the study of electromagnetic transients, the components must be represented in a wide frequency range. This introduces numerical difficulties with most of the available methods. The frequency domain identification problem is based on the estimation of a rational complex function, with real coefficients, to fit a given set of complex data. The nonlinear nature of the problem has yielded different formulations and solution methods [1-3]. Therefore, fitting techniques are necessary to develop computational models. Analysis of the frequency range considering accuracy, the shape of the frequency response, the mathematical model form and the possibility of time-domain implementation are used to decide which fitting technique is most appropriate [4]. Most techniques to fit the frequency response to rational functions use linear fitting routines [1-3] but nonlinear procedures have also been considered [5].

In this paper, to analyse the transient behaviour of air-cored transformer windings, the voltage transfer functions are calculated from the measurements which are done by network analyser. Secondly, the voltage transfer functions are fitted with rational functions by vector fitting codes and then the rational transfer functions are order-reduced by the Pade approximation. The es- timated voltages on the transformer windings using the proposed model of this paper are in good agreement with the measurements.

\section{Winding modelling}

There are mainly three methods to simulate such a transformer consisting two windings, lumped circuit model, multiconductor transmission line (MTL) model, and full-wave solution. These methods will be used in conditions where the internal behaviour of the windings is requisite. Black-box model of equipment such as a transformer is used when the output transient is needed. To achieve such a model, a frequency response analysis of such equipment is needed and via this survey, the transfer function will be obtained.

\section{Vector fitting by pole relocation}

Let us consider the rational function approximation

$$
f(s) \approx \sum_{n=1}^{N} \frac{c_{n}}{s-a_{n}}+d+s h .
$$

The residues $c_{n}$ and poles $a_{n}$ are either real quantities or come in complex conjugate pairs, while $d$ and $h$ are real. The problem under consideration is to estimate all coefficients in (1) so that a least squares approximation of $f(s)$ is obtained over a given frequency interval. We note that Eq. (1) is a nonlinear problem in terms of the unknowns, because the unknowns $a_{n}$ appear in the denominator. Vector fitting solves the problem (1) sequentially as a linear problem in two stages, both times with known poles.

\subsection{Stage No. 1 pole identification}

Let us specify a set of starting poles $\bar{a}_{n}$ in (1), and multiply $f(s)$ with an unknown function $\sigma(s)$. In addition we 
introduce a rational approximation for $\sigma(s)$. This gives the augmented problem

$$
\left[\begin{array}{c}
\sigma(s) f(s) \\
\sigma(s)
\end{array}\right] \approx\left[\begin{array}{c}
\sum_{n=1}^{N} \frac{c_{n}}{s-\bar{a}_{n}}+d+s h \\
\sum_{n=1}^{N} \frac{\tilde{c}_{n}}{s-\bar{a}_{n}}+1
\end{array}\right] .
$$

Note that in (2) the rational approximation for $\sigma(s)$ has the same poles as the approximation for $\sigma(s) f(s)$. Also, note that the ambiguity in the solution for $\sigma(s)$ has been removed by forcing $\sigma(s)$ to approach unity at very high frequencies. Multiplying the second row in (2) with $f(s)$ yields the following relation:

$$
\sum_{n=1}^{N} \frac{c_{n}}{s-\bar{a}_{n}}+d+s h \approx\left(\sum_{n=1}^{N} \frac{\tilde{c}_{n}}{s-\bar{a}_{n}}+1\right) f(s),
$$$$
(\sigma f)_{\mathrm{fit}}(s) \approx \sigma_{\mathrm{fit}}(s) f(s) \text {. }
$$

Equation (4) is linear in its unknowns $c_{n}, d, h, \tilde{c}_{n}$. Writing (3) for several frequency points gives the overdetermined linear problem $A x=b$ where the unknowns are in the solution vector $x$.

\subsection{Stage No. 2: residue identification}

In principle, we could now calculate the residues for $f(s)$ directly from Eq. (5). However, a more accurate result is in general obtained by solving the original problem in (1) with the zeros of $\sigma(s)$ as new poles $a_{n}$ for $f(s)$. This again gives an overdetermined linear problem of form $A x=b$ where the solution vector $x$ contains the unknowns $c_{n}$, d, and $h$.

$$
f(s)=\frac{(\sigma f)_{\mathrm{fit}}(s)}{\sigma_{\mathrm{fit}}(s)}=h \frac{\prod_{n=1}^{N+1}\left(s-z_{n}\right)}{\prod_{n=1}^{N}\left(s-\tilde{z}_{n}\right)} .
$$

\section{Results and discussion}

The computation of the amplitude and phase spectrum of the air-cored transformer winding takes place with 1000 measuring values, recorded in the range of $25 \mathrm{kHz}$ to $50 \mathrm{MHz}$ via Agilent $4395 \mathrm{~A}$, a network spectrum impedance analyser. The resultant transfer function includes 8 poles and 8 zeros which are given in Table.

Resultant transfer function poles and zeros.

\section{TABLE}

\begin{tabular}{c|c}
\hline \hline \multirow{3}{*}{ zeros } & $\begin{array}{c}0.1329+1.8652 \mathrm{i},-0.0947+1.7003 \mathrm{i},-0.2393+1.3827 \mathrm{i}, \\
0.2541+1.2331 \mathrm{i}, 0.1329-1.8652 \mathrm{i},-0.0947-1.7003 \mathrm{i}, \\
\end{array}$ \\
\hline \multirow{3}{*}{ poles } & $\begin{array}{c}10^{6} \times[-0.133+1.87 \mathrm{i},-0.1329-1.8652 \mathrm{i}, \\
\end{array}$ \\
& $-0.0947+1.7003 \mathrm{i},-0.0947-1.7003 \mathrm{i},-0.2393+1.3827 \mathrm{i}$, \\
&
\end{tabular}

In Fig. 1 the resultant magnitude and phase of the voltage transfer function are depicted and compared. As can be seen, there is a very acceptable correspondence between the experimental and simulation results. The investigations show that a computation of the transfer function of a transformer is possible with the presented method even in frequency ranges, which can only be approximated inadequately with methods used until now, based on equivalent circuits and corresponding impedance values. Comparison between output results of this transformer is displayed in Fig. 2 which declares good similarities between them that shows the capabilities of the proposed method.
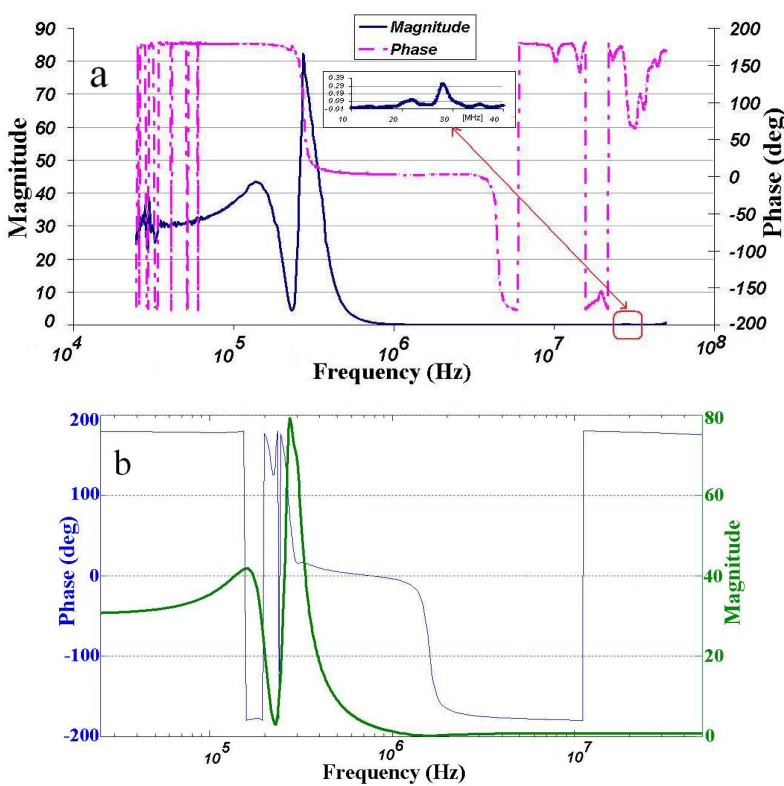

Fig. 1. Obtained transfer function (a) by experiment, (b) by vector fitting.

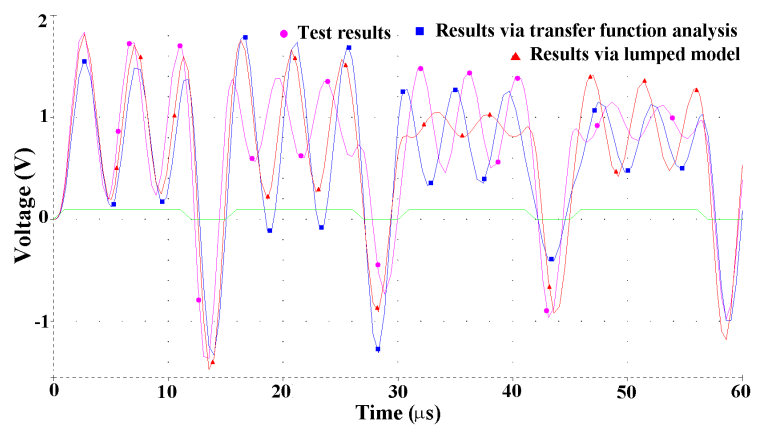

Fig. 2. Output results via test results, transfer function analysis and lumped model simulation.

\section{Conclusions}

To compute the transfer function, an analytical procedure is used which is able to detect the system parameters. A verification of the suitability of the used method takes place by the calculation of the transfer functions of a two winding air-cored transformer up to frequencies of $50 \mathrm{MHz}$. The method is suitable for an automatic calculation of transformer transfer functions without basic restrictions of the considered frequency ranges. As is described, a methodology for the estimation of a transfer 
function from frequency response measurements is presented and discussed. The transfer function parameters can be identified by the solution of an overdetermined set of equations in the least-squares sense.

\section{References}

[1] B. Gustavsen, A. Semlyen, IEEE Trans. Power Deliv. 13, 605 (1998).
[2] A.O. Soysal, A. Semlyen, IEEE Trans. Power Deliv. 8, 1627 (1993).

[3] B. Gustavsen, A. Semlyen, IEEE Trans. Power Deliv. 14, 1052 (1999).

[4] T. Henriksen, IEEE Trans. Power Deliv. 12, 372 (1997).

[5] A. Fernandes, W. Neves, in: Proc. Int. Conf. on Power Sys. Trans., 1999, p. 43. 